\title{
Measuring adverse events in pediatric inpatients with the Global Trigger Tool
}

\author{
María C. Davenporta, M.D., Paula A. Dominguez, M.D., Juan P. Ferreira ${ }^{a}$, M.D., \\ Ana L. Kannemann ${ }^{a}$, M.D., Agustina Paganini ${ }^{a}$, M.D. and Fernando A. Torres ${ }^{a}$, M.D.
}

\begin{abstract}
Introduction: The safety of inpatients is a priority in the health care system. The Global TriggerTool seems to be suitable to estimate the incidence of adverse events (AE) in pediatric inpatients. Objectives: To describe the incidence and categories of AE in pediatric inpatients using the Global Trigger Tool and to identify risk factors associated to their development.

Population and methods: Retrospective study. Medical records of 200 patients hospitalized at Hospital Elizalde during 2013 were included. Outcome measures: number of AE/100 admissions and distribution of harm. A chi ${ }^{2}$ test, Student's t test and Pearson's correlation test were carried out. Significance level $=p<0.05$. Results: The study detected 289 triggers (1.4/ patient); 52 AEs (26 AEs/100 patients, 95\% CI: 20.4-32.5). There was at least one AE every 36 patients; 7 patients had more than one AE; 45 AEs were in the $\mathrm{E}$ and $\mathrm{F}$ categories (temporary harm). Medical care triggers were associated to AEs (OR 8.1; 95\% CI: 3.7-17.3, $p<0.001$ ). A positive correlation was found between the number of triggers and the number of AEs per patient $(\mathrm{R}=0.46 ; p<0.001)$. Being hospitalized in a closed unit (OR 2.8; 95\% CI: 1.2-6.5; $p=0.03$ ) and a longer hospital stay were associated to AEs $(p<0.001)$.

Conclusion: An AE frequency of 26\% was identified, and most AEs resulted in temporary harm. The presence of AEs was associated to hospitalization in a closed unit, longer hospital stay, higher number of triggers and general care triggers.

Key words: patient safety, medical error, indicator of healthcare quality.
\end{abstract}

http:/ / dx.doi.org/10.5546/ aap.2017.eng.357

To cite: Davenport MC, Domínguez PA, Ferreira JP, et al. Measuring adverse events in pediatric inpatients with the Global Trigger Tool. Arch Argent Pediatr 2017;115(4):357-363.

This study received a research fellowship from the Subcommittee for Scholarships and Awards of the Sociedad Argentina de Pediatría (Argentine Society of Pediatrics, 2015).

Conflict of interest: None.

Received: 7-10-2016 Accepted: 1-24-2017 implemented to detect and reduce harm, and to improve the quality of care. $^{1,2}$

AEs incidence in adult inpatients differs, according to the literature, from $3 \%$ to $16 \%$. Children are more vulnerable to AEs, mainly because there is an increased risk of medication errors, with a reported frequency of $11 \%$. $^{3,4}$

Moreover, the incidence of AEs related to medical care and medication errors varies with the method used for their detection. ${ }^{5,6}$ The Global Trigger Tool (GTT) seems to be the best to detect AEs, both in the adult and in the pediatric population, with a sensitivity of $94 \%$ and a specificity of $100 \%{ }^{7}$

The GTT was developed by the Institute for Healthcare Improvement, in order to identify AEs and measure their incidence rates. It consists of a retrospective assessment of a random sample of medical records to find triggers and identify potential AEs. These triggers are organized in 6 modules related to medical care, medication, surgery, emergency, intensive care and perinatal-obstetric. ${ }^{7}$

The authors of the GTT found 36.7 AEs every 100 patients and 76.3 AEs every 1000 patients/day. Most AEs resulted in temporary harm. This number was almost three times higher than the values published so far, indicating that this tool may be useful and sensitive enough to identify AEs.?

Although several healthcare policies have been implemented in recent years to increase patients' safety, there is no accurate data on this issue at a local level, in particular, in the population of children hospitalized at Hospital General de Niños Pedro de Elizalde (HGNPE). Determining the frequency and 
distribution of AEs related to medical care would be useful to develop strategies aimed at reducing harm.

The objectives of this study were to describe the incidence of AEs and their categories in pediatric inpatients, using the GTT, and to identify AE related risk factors.

\section{POPULATION AND METHODS}

Design: Retrospective study. Medical records of patients hospitalized at the HGNPE during at least 48 hours in 2013 (January 1 $^{\text {st }}$ - December $31^{\text {st }}$ ) were randomly included. Medical records had to be complete, their pages numbered, and had to include a discharge summary and be accurately coded. If the medical record selected described more than one hospitalization during the study year, the most recent hospitalization was considered. Medical records belonged to patients hospitalized in multipurpose and closed units (Pediatric ICU and Neonatology Division).

Medical records of patients hospitalized due to psychiatric disorders and social reasons were excluded.

Medical records were systematically screened for AEs using the GTT. This tool includes 52 preset triggers, organized in 6 modules (cares, medication, surgical, emergency, intensive care and perinatal-obstetric) to identify possible causes of healthcare-related harm. A trigger is an element that is present in the medical record and that may be associated to the presence of an $\mathrm{AE}$, and requires reviewers to investigate further into such record (see the Annex).

The hospital has no maternity unit; therefore, the triggers in the perinatal-obstetric module (applicable to pregnant women) did not apply to our population.

Medical records were reviewed in depth (progress reports, medication, lab reports, surgical reports, nurse sheets, consultation records, etc.).

Reviewers underwent the same training and were the same along the study. Each reviewer received 7-10 medical records per week and afterwards medical records were exchanged. In case of discrepancy, they were evaluated by a third reviewer.

If at least one trigger was found, the record was reviewed more thoroughly to search for an associated AE. If found, the event was categorized. If no trigger was found, the review of that medical record was concluded. This procedure was in place until the required sample size was achieved. The observation of medical records was completed in 7 months.

AEs were defined as unintended physical injury resulting from or contributed to by medical care that requires additional monitoring, treatment or hospitalization, or that results in death. ${ }^{8}$

The study outcome measure was the number of AEs/100 admissions. Harm resulting from the AE was classified into 5 categories: temporary harm requiring intervention (category E), temporary harm requiring a prolonged hospitalization $(F)$, permanent harm (G), permanent harm requiring specific intervention to sustain life $(\mathrm{H})$ and death $(\mathrm{I}){ }^{9}$

Outcome measures to be controlled were age, sex, total hospital length of stay, length of stay at the general care unit, ICU and Neonatology Unit, and diagnosis that led to the hospitalization.

\section{Statistical analysis}

Categorical outcome measures were expressed as percentages with $95 \%$ confidence intervals. Numerical outcome measures were described using an average and a deviation.

To estimate the incidence of AEs using the GTT, the total number of AEs was used on the total number of patients and was multiplied by 100 to express it as a percentage.

The degree of inter-observer agreement was evaluated for the observation of triggers and AEs by means of the Kappa coefficient.

The $t$ test was used to determine the association between the mean value of triggers per patient and the presence of AEs. The chi ${ }^{2}$ test was used to assess if there was an association between the presence of AEs and the place where they occurred, the kind of harm and the site, and the kind of trigger and the presence of an AE. The correlation between the number of triggers and AEs was measured using Pearson's coefficient. A significance level of $p<0.05$ was adopted (SPSS 11.1).

\section{Sample size and sample selection}

Out of 10,000 hospital discharges every year, a sample size of 200 medical records was calculated, based on an AE frequency of $15 \% \pm 5 \%$, for a $95 \%$ confidence interval and considering a simple random sampling (Epi Info 7.1). ${ }^{3}$

Sampling was carried out through simple randomized selection from the medical records repository. If the selected medical record was not found, or if it did not meet the inclusion criteria or met any of the exclusion criteria, the next record was immediately used. 


\section{Ethical considerations}

Since this was a retrospective study on the analysis of medical records, it was not considered necessary to obtain an informed consent. Data were recorded keeping patients' identities anonymous.

The study was approved by the Research and Teaching Committee and the Bioethics Committee of the HGNPE (file no. 782014).

\section{RESULTS}

Medical records of 211 patients hospitalized at the HGNPE were reviewed. Five (5) records were excluded because they were incomplete and 6 because a discharge summary had not been included. The final sample consisted of 200 medical records corresponding to a total of 1690 days of hospitalization. All records were reviewed by 2 investigators and there was a discrepancy as regards the presence of a trigger in 12 records, which were evaluated by a third investigator.

Table 1 shows the main characteristics of the population.

The reasons for hospitalization were; firstly, the diagnosis of acute low respiratory tract infection; secondly, non-respiratory infections (cellulites, abscess, acute diarrhea, urinary tract infection, febrile neutropenia); thirdly, surgical causes (acute abdomen); and, lastly, other diagnoses (convulsive syndrome, apparent life-threatening event, onset of oncological disease, trauma) (Table 1).
Two hundred and eighty nine triggers (1.4/ patient) were detected and 52 AEs were identified (26 AEs / 100 patients, 95\% CI: 20.4- 32.5; and 30.7 AEs / 1000 hospitalization days, 95\% CI: 23.5-40.1). Eighty two (82) medical records did not have triggers nor AEs. Out of the remaining 118 records most included 1 trigger (range 1 to 16). Thirty six (36) patients had at least one AE and 7 patients had more than one (2-8 AEs).

Forty five percent (45\%) (95\% CI: 38.4-49.6) of triggers corresponded to the general medical care module; $27 \%$ (95\% CI: 21.8-31.8) to the medication module; $16 \%$ (95\% CI: 13.8-19.1) to triggers related to the emergency department and the ICU; and the remaining 10\% (IC 95\%: 8.2-12.7), to the surgical module (Table 2).

Sixteen percent $(16 \%)(n=21)$ of triggers associated to the module of medical care were identified by the item "other triggers", which mostly corresponded to the description of phlebitis, infiltration of the peripheral line and pneumothorax secondary to surgical procedures. These situations were not described in the GTT (Table 2).

Eighty three percent $(83 \%)(n=43)$ of AEs were identified based on triggers under the medical care module; $11 \%(n=6)$, under the surgical module; $4 \%(\mathrm{n}=2)$ under the medication module; and the remaining $2 \%(\mathrm{n}=1)$, under the emergency department and pediatric ICU module (Table 2).

TABLE 1. Main characteristics of the population. Medical records reviewed, $N=200$

\begin{tabular}{|c|c|c|c|c|}
\hline & Total $(\%)$ & Mean & SD & $95 \% \mathrm{CI}$ \\
\hline Age in months & & 52.2 & 60.9 & $43.7-60.6$ \\
\hline Male & $102(51 \%)$ & & & $44.2-57.4$ \\
\hline Female & $98(49 \%)$ & & & $42.1-55.9$ \\
\hline \multicolumn{5}{|l|}{ Hospital length of stay in days } \\
\hline Total & 1690 & 8.45 & 11.6 & 7.9-9.1 \\
\hline - Multipurpose Unit & 1355 & 7.13 & 7.08 & $6.7-7.5$ \\
\hline$-\mathrm{ICU}$ & 196 & 11.5 & 21.5 & $10.3-12.6$ \\
\hline - Neonatology Unit & 139 & 15.4 & 13.8 & $13.1-17.7$ \\
\hline \multicolumn{5}{|l|}{ Place of hospitalization } \\
\hline - Multipurpose Unit & $174(87 \%)$ & & & $81.6-90.9$ \\
\hline - ICU & $17(8.5 \%)$ & & & 5.3-13.1 \\
\hline - Neonatology Unit & $9(4.5 \%)$ & & & $2.3-8.3$ \\
\hline \multicolumn{5}{|l|}{ Diagnosis at admission } \\
\hline - ALRTI & $72(36 \%)$ & & & $29.6-42.8$ \\
\hline - Source of infection & $52(26 \%)$ & & & $20.4-32.5$ \\
\hline - Reason for the surgery & $48(24 \%)$ & & & $18.6-30.4$ \\
\hline - Other & $28(14 \%)$ & & & 9.8-19.5 \\
\hline
\end{tabular}

SD: standard deviation; CI: confidence interval; ALRTI: acute low respiratory tract infection; ICU: Intensive Care Unit. 
Adverse events detected per GTT module and the severity of harm are described in Table 3.

Nosocomial infections accounted for $70 \%$ of detected AEs.

There was an adequate inter-observer agreement for the identification of triggers and AEs (kappa 0.56, $p=0.01$; kappa 0.96, $p=0.01$ ).

When evaluating the association between the number of triggers per patient and the presence of AEs, it was observed that the mean number of triggers in the group of patiens who did not experience any AEs was 0.8 , in contrast with $4.3(p<0.001)$ in the group with at least one AE. Moreover, a positive correlation was found between the number of triggers and the number of AEs per patient (Pearson's correlation $\mathrm{R}=0.46$; $p<0.001$ ).

Triggers associated to general medical care showed a significant association with AEs when compared to other triggers: $43 / 131$ vs. $9 / 158$ (OR: 8.1; $95 \%$ CI: 3.7-17.3, $p<0.001$ ).

With respect to the place of hospitalization, it was observed that $10 / 26$ patients hospitalized in a closed unit experienced AEs, compared to 24/174

TABLE 2. Triggers detected and associated adverse events

\begin{tabular}{|c|c|c|}
\hline Adverse event module & No. of triggers & No. of adverse events \\
\hline \multicolumn{3}{|l|}{ Medical care } \\
\hline C1: Transfusion or use of blood products & 15 & 0 \\
\hline C2: Code, cardiac or pulmonary arrest, or rapid response team activation & 6 & 1 \\
\hline C4: Positive blood culture & 15 & 2 \\
\hline C5: Emboli or deep vein thrombosis & 2 & 1 \\
\hline C6: Decrease in hemoglobin or hematocrit $>25 \%$ & 6 & 0 \\
\hline C7: Accident/fall / injury in the hospital & 1 & 0 \\
\hline C8: Pressure ulcers & 1 & 1 \\
\hline C9: Readmission within 30 days & 33 & 7 \\
\hline C11: Nosocomial infections & 28 & 28 \\
\hline C14: Any procedural complication & 3 & 0 \\
\hline C15: Other & 21 & 3 \\
\hline Total of cares & 131 & 43 \\
\hline \multicolumn{3}{|l|}{ Medication } \\
\hline M2: QUICK > 100 seconds & 3 & 0 \\
\hline M4: Glucose $<50 \mathrm{mg} / \mathrm{dL}$ & 3 & 0 \\
\hline M5: Rising BUN or serum creatinine greater than two times $(2 x)$ baseline & 4 & 0 \\
\hline M6: Vitamin K administration & 2 & 0 \\
\hline M7: Diphenhydramine use & 29 & 0 \\
\hline M10: Anti-emetic use & 20 & 0 \\
\hline M11: Over-sedation/hypotension & 3 & 0 \\
\hline M12: Abrupt medication stop & 13 & 0 \\
\hline M13: Other & 2 & 2 \\
\hline Total de medication & 79 & 2 \\
\hline \multicolumn{3}{|l|}{ Surgical } \\
\hline S1: Return to surgery & 4 & 0 \\
\hline S2: Change in procedure & 4 & 0 \\
\hline S3: Admission to ICU & 7 & 0 \\
\hline S4: Reintubation in the Operating Room & 4 & 1 \\
\hline S5: Intra-operative $x$-ray & 1 & 0 \\
\hline S7: Mechanical ventilation greater than 24 hours post-op & 4 & 0 \\
\hline S8: Inotropics, naloxone or flumazenil & 1 & 0 \\
\hline S11: Other & 6 & 5 \\
\hline Total surgical & 31 & 6 \\
\hline \multicolumn{3}{|l|}{ Emergency/ICU/perinatal-obstetric } \\
\hline E1: Readmission to ED within 48 hours & 21 & 0 \\
\hline E2: Time in ED greater than 6 hours & 17 & 0 \\
\hline I1: Nosocomial pneumonia & 2 & 1 \\
\hline I2: Readmission to the ICU & 2 & 0 \\
\hline I4: Intubation/reintubation & 6 & 0 \\
\hline Total Emergency/ICU/perinatal & 48 & 1 \\
\hline Total & 289 & 52 \\
\hline
\end{tabular}

ICU: Intensive Care Unit. 
patients hospitalized only in a multipurpose unit (OR 2.8; 95\% CI: 1.2-6.5, $p=0.03$ ).

The average hospital length of stay was higher in patients with AEs than in those who did not experience AEs (21.1 vs. 5.7, respectively; $p=0.001$ ).

No association was found between the type of harm and the place where it occurred. In addition, no association was noticed between the age or the diagnosis and an AE.

\section{DISCUSSION}

In our study, using the GTT, a frequency of $26 \%$ of AEs was observed in hospitalized patients. An association was found between the number of triggers, hospitalization in a closed unit and hospital length of stay, and the presence of AEs. About half of the triggers found were under the general medical care module. Most of the AEs corresponded to temporary harm to the patient.

The frequency observed of AEs was lower than that reported by Kirkendall with the same tool $(36 \%)$. Moreover, in the same study, the number of triggers per patient was slightly lower than that reported in our study (1.4 vs. 1.7). ${ }^{7}$ This difference may be attributed to the type of medical records and coding (manual in our case and electronic in the study published by Kirkendall). The electronic medical record may be more specific and sensitive to detect triggers. ${ }^{10}$

Stockwell described an AE frequency of $40 \%$ using the Pediatric All-Cause Harm Measurement Tool (PACHMT), a specific tool for pediatric use based on the GTT.

This difference in frequency with respect to our study may be due to the fact that the new tool has even more sensitive triggers to detect AEs in the pediatric population, a greater complexity of the study population or a potential different interpretation of AE. ${ }^{11}$
Eighty three percent $(83 \%)$ of AEs were identified based on triggers belonging to the medical care module of the GTT, which was partially consistent with Kinkerdall who observed that $95 \%$ of the AEs detected were associated to medical care and medication modules. ${ }^{7}$ In both studies, the possible cause of this high percentage is that main triggers -such as nosocomial infections-, are also considered AEs on their own.

Our study showed that the greater the number of triggers per patient, the higher the possibility of detecting an AE; this was in line with findings reported by other authors. ${ }^{12,13}$ This last finding may be the starting point for future investigations aimed at evaluating what combinations of triggers have a closer association with AEs, or a better chance of predicting them.

With respect to harm distribution, most AEs were in categories F and E. However, in certain cases harm was permanent or required an intervention to sustain life ( $>10 \%)$, a percentage that was remarkably different to that reported by Kirkendall, ${ }^{7}$ who found only $2 \%$. Nevertheless, other authors also reported values closer to our findings: Stockwell ${ }^{11}(10.4 \%)$ and Naessens ${ }^{12}(8 \%)$.

The most frequent AE was nosocomial infection. Other authors also described infiltration of venous catheter, hypoglycemia, pressure ulcers and procedural complications. . $^{11,14,15}$

Nosocomial infections require specific management within the concept of medical errors and AEs. There are protocols in place for their monitoring and the reduction of their incidence.

In line with other studies, AEs were more frequent in closed units. This is probably linked to variables such as prematurity, unstable clinical condition and prescription errors. ${ }^{16-18}$

Our study observed a significant association between the presence of AEs and total length

TABLE 3. Adverse events detected per Global Trigger Tool module and severity of harm

\begin{tabular}{|c|c|c|c|c|c|c|}
\hline $\begin{array}{l}\text { Severity of harm } \\
\text { Modules }\end{array}$ & All categories & $\mathrm{E}$ & $\mathbf{F}$ & G & $\mathbf{H}$ & I \\
\hline Medical care & $43(83 \%)$ & 17 & 22 & 1 & 2 & 1 \\
\hline Medication & $2(4 \%)$ & 1 & 1 & & & \\
\hline Surgical & $6(11 \%)$ & 3 & 1 & & 2 & \\
\hline Emergency/ICU & $1(2 \%)$ & & & & 1 & \\
\hline Total (n) & 52 & 21 & 24 & 1 & 5 & 1 \\
\hline$\%$ & $100 \%$ & $40 \%$ & $46 \%$ & $1.9 \%$ & $9.6 \%$ & $1.9 \%$ \\
\hline $95 \% \mathrm{CI}$ & & $28.1-53.9$ & $33.3-59.5$ & $0.34-10.1$ & $4.1-20.6$ & $0.34-10.1$ \\
\hline
\end{tabular}

ICU: Intensive Care Unit; E: requires intervention; F: prolonged hospitalization; G: permanent hospitalization;

$\mathrm{H}$ : permanent to sustain life; I: death; CI: confidence interval. 
of stay -as reported by Härkänen and Najjar-, probably related to complex disorders, comorbidity and multiple medications. ${ }^{19,20}$ However, Rutberg, based on a similar finding, suggested that AEs could be the cause and not the consequence of prolonged hospitalizations. ${ }^{15}$

Nineteen percent $(19 \%)$ of AEs found were detected by "other triggers", which reflected the possibility that some AEs that are frequent in the pediatric population were underrepresented by specific GTT triggers. AEs related to peripheral venous lines, such as phlebitis and infiltration, and pneumothorax secondary to surgical procedures, are some examples of triggers that are not included in the GTT. Their inclusion in the future may improve the tool's AE detection capacity.

This study has some limitations that should be considered. Firstly, the sample size selected was aimed to determine the incidence of AEs. In this sense, the goal was achieved; however, some associations could not be confirmed. This may be attributed to a beta error effect, which could have been avoided had the sample size been larger.

Secondly, observers were investigator physicians trained in the use of the GTT, but they were not specialized in the screening of triggers and AEs. As a consequence, the recommendation of using two observers was adopted, with a third observer in case of discrepancies.

This study also shows some strengths that should be noted. A simple random sampling method was used, which is the best strategy to control unknown confounding variables. Moreover, the studied population had similar characteristics to the population hospitalized at our facility. Finally, this study uses the GTT, one of the most sensitive and specific tools to identify AEs. It offers a user friendly method to evaluate the incidence of AEs in hospitalized patients. Results of this study could contribute with valuable data on the frequency of AEs experienced by our population, which would facilitate the design and implementation of strategies to improve the quality of care in specific areas of our facilities. Further research on this topic is needed.

\section{CONCLUSIONS}

The AE frequency was $26 \%$ and most AEs resulted in temporary harm. Only the number of triggers per patient, hospitalization in a closed unit and total hospital length of stay were associated to the presence of AEs.

\section{REFERENCES}

1. Steering Committee on Quality Improvement and Management and Committee on Hospital Care. Policy statement-principles of pediatric patient safety: reducing harm due to medical care. Pediatrics 2011;127(6):1199-210.

2. Institute of Medicine. To err is human: building a safer health system. Washington DC: National Academies Press; 2000.

3. Matlow AG, Cronin CM, Flintoft V, et al. Description of the development and validation of the Canadian Paediatric Trigger Tool. BMJ Qual Saf 2011;20(5):416-23.

4. Otero P, Leyton A, Mariani G, et al. Medication errors in pediatric inpatients: prevalence and results of a prevention program. Pediatrics 2008;122(3):e737-43.

5. Franklin BD, Birch S, Schachter M, et al. Testing a trigger tool as a method of detecting harm from medication errors in a UK hospital: a pilot study. Int J Pharm Pract 2010;18(5): 30511.

6. Takata GS, Mason W, Taketomo C, et al. Development, testing and findings of a pediatric-focused trigger tool to identify mediation-related harm in US children's hospitals. Pediatrics 2008;121(4):e927-35.

7. Kirkendall ES, Kloppenborg E, Papp J, et al. Measuring adverse events and levels of harm in pediatric inpatients with the Global Trigger Tool. Pediatrics 2012;130(5):e120614.

8. Griffin FA, Resar RK. IHIGlobal Trigger Tool for Measuring Adverse Events. 2nd ed. Cambridge:Institute for Healthcare Improvement; 2009.

9. National Coordinating Council for Medication Error Reporting and Prevention. Types of Medication Error [Internet]. [Accessed on: January 30, 2017.] Available at: http: / / www.nccmerp.org/types-medication-errors.

10. Murff HJ, Forster AJ, Peterson JF, et al. Electronically screening discharge summaries for adverse medical events. J Am Med Inform Assoc 2003;10(4):339-50.

11. Stockwell DC, Bisarya H, Classen DC, et al. A trigger tool to detect harm in pediatric inpatient settings. Pediatrics 2015;135(6):1037-42.

12. Naessens JM, O’Byrne TJ, Johnson MG, et al. Measuring hospital adverse events: assessing inter-rater reliability and trigger performance of the Global Trigger Tool. Int J Qual Health Care 2010;22(4):266-74.

13. Hwang JI, Chin HJ, Chang YS. Characteristics associated with the occurrence of adverse events: a retrospective medical record review using the Global Trigger Tool in a fully digitalized tertiary teaching hospital in Korea. J Eval Clin Pract 2014;20(1):27-35.

14. Nilsson L, Pihl A, Tågsjö M, et al. Adverse events are common on the intensive care unit: results from a structured record review. Acta Anaesthesiol Scand 2012;56(8):959-65.

15. Rutberg H, Borgstedt Risberg M, Sjödahl R, et al. Characterisations of adverse events detected in a university hospital: a 4-year study using the Global Trigger Tool method. BMJ Open 2014;4(5):e004879.

16. Kaushal R, Bates DW, Landrigan C, et al. Medication errors and adverse drug events in pediatric inpatients. JAMA 2001;285(16):2114-20.

17. Serra VV, Pena F, Ossorio MF, et al. Errores de prescripción de drogas endovenosas en una unidad de cuidados neonatales de la ciudad de Buenos Aires. Revista Facultad de Ciencias Médicas 2012;69(1):15-9.

18. Chedoe I, Molendijk HA, Dittrich ST, et al. Incidence and nature of medication errors in neonatal intensive care with strategies to improve safety: a review of the current literature. Drug Saf 2007;30(6):503-13.

19. Härkänen M, Kervinen M, Ahonen J, et al. Patient-specific 
risk factors of adverse drug events in adult inpatients -evidence detected using the Global Trigger Tool method. J Clin Nurs 2015;24(3-4):582-91.
20. NajjarS,Hamdan M,Euwema MC, etal. The Global Trigger Tool shows that one out of seven patients suffers harm in Palestinian hospitals: challenges for launching a strategic safety plan. Int J Qual Health Care 2013;25(6):640-7. 
ANNEX

Global Trigger Tool - Triggers

\section{Adverse event modules}

General care (C)

C1: Transfusion or use of blood products

C2: Code, cardiac or pulmonary arrest, or rapid response team activation

C3: Dialysis

C4: Positive blood cultures

C5: X-Ray or Doppler studies for emboli or deep vein thrombosis

C6: Decrease in hemoglobin or hematocrit $>25 \%$

C7: Accident/fall/injury in the hospital

C8: Pressure ulcers

C9: Readmission within 30 days

C10: Physical restraint

C11: Nosocomial infections

C12: In-Hospital stroke

C13: Transfer to higher level of care

C14: Any procedural complication

C15: Others (the name of the trigger should be filled in here)

\section{Medication (M)}

M1: Clostridium difficile positive culture

M2: QUICK > 100 seconds

M3: INR $>6$

M4: Glucose $<50 \mathrm{mg} / \mathrm{dL}$

M5: BUN or serum creatinine greater than two times $(2 x)$ baseline

M6: Vitamin K administration

M7: Diphenhydramine use

M8: Flumazenil use

M9: Naloxone use

M10: Anti-emetic use

M11: Over-sedation/hypotension

M12: Abrupt medication stop

M13: Other (medication trigger module)

\section{Surgical (S)}

S1: Return to surgery

S2: Change in surgical procedure

S3: Admission to the Pediatric Intensive Care Unit

S4: Intubation or reintubation at the operating room or recovery unit

S5: Intra-operative $x$-ray or in the recovery unit

S6: Intra- or postoperative death

S7: Mechanical ventilation greater than 24 hours postoperatively

S8: Intra-operative use of inotropics, naloxone or flumazenil

S9: Postoperative Increase in troponin levels greater than $1.5 \mathrm{ng} / \mathrm{mL}$

S10: Organ injury, repair or removal

S11: Any surgical complication

\section{Emergency (E), pediatric ICU (I) and perinatal-obstetric (P)}

E1: Readmission within 48 hours

E2: Time in ED $>6$ hours

I1: Nosocomial pneumonia

I2: Readmission to the ICU

I3: In-Unit procedure

I4: Intubation/reintubation

P1: Use of terbutaline

P2: 3rd- or 4th-Degree lacerations

P3: Platelet count $<50000 / \mathrm{mL}$

P4: Estimated blood loss > $500 \mathrm{~mL}$ (vaginal) or $>1000 \mathrm{~mL}$ (c-section)

P5: Specialty consult

P6: Oxytocic agents

P7: Instrumented delivery

INR: International Normalized Ratio. 